\title{
Analysis on Influencing Factors of Blasting Vibration Caused by Shallow Tunnel Excavation
}

\author{
Haixia Wei ${ }^{1}$, Zheng Qu ${ }^{1}$, Jie Zhu ${ }^{1, ~ *, ~ Q i a n g q i a n g ~ Z h a n g ~}{ }^{1,2}$ \\ ${ }^{1}$ School of Civil Engineering, Henan Polytechnic University, Jiaozuo, China \\ ${ }^{2}$ School of Civil Engineering, Central South University, Changsha, China
}

\section{Email address:}

whx19820828@163.com (Haixia Wei),42065795@qq.com (Zheng Qu),108452037@qq.com (Jie Zhu), 1075328886@qq.com (Qiangqiang Zhang)

*Corresponding author

\section{To cite this article:}

Haixia Wei, Zheng Qu, Jie Zhu, Qiangqiang Zhang. Analysis on Influencing Factors of Blasting Vibration Caused by Shallow Tunnel Excavation. American Journal of Civil Engineering. Vol. 7, No. 4, 2019, pp. 108-112. doi: 10.11648/j.ajce.20190704.14

Received: September 8, 2019; Accepted: October 17, 2019; Published: October 23, 2019

\begin{abstract}
With the widespread construction of urban subway, more and more shallow tunnels will be constructed by blasting, and the problem of blasting vibration will become more prominent. Because of the randomness and variability of blasting source and topographic and geological factors, the propagation mechanism and influencing factors of blasting vibration wave are very complex. Based on blasting-vibration sample data obtained from the established numerical model of blasting excavation in shallow tunnel, the relational degree analysis was carried out for influencing factors of blasting vibration caused by shallow tunnel excavation with method of grey relational analysis. The results of the study are as follows: Among the four related factors, there is no optimal factor, and the maximum charge of one section is the quasi-optimal factor; The charge of the first section is the main factor affecting the peak particle velocity of blasting vibration; The maximum charge of one section is the main factor affecting the main frequency of blasting vibration; The delay interval is the main factor affecting the duration of blasting vibration. Furthermore, the measures to control blasting vibration caused by shallow tunnel excavation were put forward, such as reducing the charge of the first section, reducing the maximum charge of one section and rationally setting up the delay interval. The study has important guiding significance for safe blasting construction in shallow tunnel and scientific control of blasting vibration effect.
\end{abstract}

Keywords: Shallow Tunnel, Method of Grey Relational Analysis, Blasting Vibration, Influencing Factors, Control Measures

\section{Introduction}

The subway has been opened in 33 cities in China up to now. In the large-scale development of urban-subway construction, it is inevitable that more and more shallow tunnels will be constructed through the urban areas with many buildings on them. As the most economical and effective excavation method of rock mass engineering, borehole blasting is also the main construction method of rock tunnels. Compared with other blasting projects, the ground surface often has high blasting vibration intensity because of close distance from the blasting source in the blasting construction in shallow tunnels. Blasting vibration caused by underground excavation has become a hot and difficult research topic in the field of blasting engineering in recent years [1-7]. Because of the randomness and variability of blasting source and topographic and geological factors, the generation and propagation mechanism of blasting vibration wave is very complex. Although a lot of research has been done on the influence factors of blasting vibration and the characteristics of blasting vibration wave [8-13], there are still some problems that have not been fundamentally solved.

Based on blasting-vibration sample data obtained from the established numerical model of blasting excavation in shallow tunnel, the relational degree analysis was carried out for influencing factors of blasting vibration caused by shallow tunnel excavation with method of grey relational analysis. Furthermore, the measures to control blasting vibration caused by shallow tunnel excavation were put forward. The 
study has important guiding significance for safe blasting construction in shallow tunnel and scientific control of blasting vibration effect.

\section{Principle of Method of Grey Relational Analysis}

Method of grey relational analysis is a method to measure the degree of relation among factors according to the similarity or difference of the development trend of factors [14-16]. Method of grey relational analysis has been widely used in industry, agriculture, military, economy, ecology and other fields because of the ability to deal with the grey system with incomplete information, which has relatively high accuracy in the evaluating irregular indicators with small samples.

The specific calculation steps of method of grey relational analysis are as follows [17]:

Step 1: Determine the analysis sequence.

The reference sequence reflecting the behavior characteristics of the system and the comparison sequence affecting the behavior characteristics of the system are determined.

Let the reference sequence $Y=\{Y(k) \mid k=1,2, \cdots, n\}$, the comparison sequence $X_{i}=\left\{X_{i}(k) \mid k=1,2, \cdots, n\right\}$, where $i=1,2, \ldots, m$.

Step 2: Make variables dimensionless.

The data in each factor sequence in the system may be different in dimension, and it is not convenient to compare or it is difficult to get the correct conclusion in comparison. Therefore, the dimensionless processing of data is generally required in the analysis of grey relational degree.

$$
x_{i}(k)=\frac{X_{i}(k)}{X_{i}(l)}, k=1,2, \cdots, n ; i=0,1,2, \cdots, m
$$

Step 3: Calculate the relational coefficient.

The relational coefficient of $x_{0}(k)$ and $x_{i}(k)$ :

$$
\xi_{i}(k)=\frac{\min _{i} \min _{k}\left|y(k)-x_{i}(k)\right|+\rho \max _{i} \max _{k}\left|y(k)-x_{i}(k)\right|}{\left|y(k)-x_{i}(k)\right|+\rho \max _{i} \max _{k}\left|y(k)-x_{i}(k)\right|}
$$

Let $\triangle_{i}(k)=\left|y(k)-x_{i}(k)\right|$, then

$$
\xi_{i}(k)=\frac{\min _{i} \min _{k} \triangle_{i}(k)+\rho \max _{i} \max _{k} \triangle_{i}(k)}{\triangle_{i}(k)+\rho \max _{i} \max _{k} \triangle_{i}(k)}
$$

where $\rho$ is the resolution coefficient, $\rho \in(0, \infty)$. The smaller $\rho$ is, the greater the resolution is. The general range of $\rho$ is $(0,1)$. When $\rho \leq 0.5463$, the resolution is the best, usually $\rho=0.5$.

Step 4: Calculate the relational degree.

Because the relational coefficient is the value of the relational degree between the comparison sequence and the reference sequence at each time, it has more than one number, and the information is too scattered to make overall comparison conveniently. It is necessary to concentrate the relational coefficient at each time into one value, that is, to find its average value as a quantitative expression of the relational degree between the comparison sequence and the reference sequence.

The formula of $r_{i}$ is as follows:

$$
r_{i}=\frac{1}{n} \sum_{k=1}^{n} \xi_{i}(k), k=1,2, \cdots, n
$$

Step 4: Rank the relational degree.

The relational degree is sorted by size. If $r_{1}<r_{2}$, the reference sequence $y$ is more similar to the comparison sequence $x_{2}$.

After calculating the relational coefficient between sequence $X_{i}(k)$ and $Y(k)$, the average value of relational coefficients is calculated. The average $r_{i}$ is called the relational degree between $Y(k)$ and $X_{i}(k)$.

\section{Analysis on Influencing Factors of Blasting Vibration Caused by Shallow Tunnel Excavation}

\subsection{Determination of System Characteristic Variables and Related Factor Variables}

The three main factors of blasting vibration, i.e. particle peak velocity, main frequency and duration, are often taken as the indexes to measure the damage degree of blasting vibration. Because the sample data in this paper are taken from numerical simulation experiments, the average combined velocity and velocity attenuation ratio in a given period of time are selected to represent the duration of blasting vibration in order to shorten the running time of the computer. When analyzing blasting vibration caused by shallow tunnel excavation with method of grey relational analysis, particle peak velocity, main frequency, average combined velocity and velocity attenuation rate were selected as system characteristic variables, and were recorded as $Y_{1}, Y_{2}, Y_{3}$ and $Y_{4}$ respectively.

There are many factors affecting blasting vibration, including total charge, maximum charge of one section, maximum charge in cut section, minimum delay interval, number of sections, distance from blasting centers, number of free surfaces, hole network parameters, geological conditions, etc. The vibration effect produced by cutting blasting in shallow tunnel is the most obvious in practical engineering. Therefore, the excavation process of cutting blasting was simulated by numerical simulation test. Regardless of the factors that can not be quantitatively described, under the condition of constant distance from blasting centers and total charge, the four factors of charge of the first section, maximum charge of one section, number of sections and delay interval were selected as the relevant variables for analysis, which were recorded as $X_{1}, X_{2}, X_{3}$ and $X_{4}$ respectively. 


\subsection{Acquisition of Sample Data of Blasting Vibration Caused by Shallow Tunnel Excavation}

Using numerical software LS-DYNA, the finite element model of cut blasting in shallow tunnel was established, as shown in Figure 1. 3994 is the number of selected surface feature points, from which sample data were obtained. The size of the model is $50 \mathrm{~m} \times 30 \mathrm{~m} \times 24 \mathrm{~m}$, and the length of the tunnel face without excavation is $24 \mathrm{~m}$. Charge diameter is 52 $\mathrm{mm}$, length is $400 \mathrm{~mm}$, mud plugging is $400 \mathrm{~mm}$, single hole charge is $0.85 \mathrm{Kg}$.

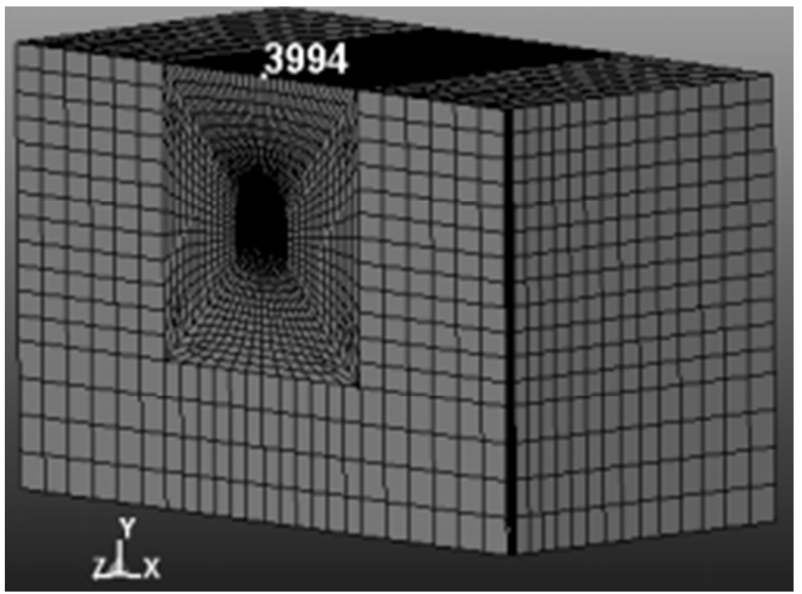

Figure 1. Finite element model of cutting blasting in shallow tunnel.

The explosive material model is defined by JWL state equation:

$$
P_{c j}=A\left(1-\frac{\omega}{R_{1} V}\right) e^{-R_{1} V}+B\left(1-\frac{\omega}{R_{2} V}\right) e^{-R_{2} V}+\frac{\omega E}{V}
$$

where $A, B, R_{1}, R_{2}, \omega$ are input parameters, $A=371.2 \mathrm{Gpa}$, $B=3.231 \mathrm{Gpa}, R_{l}=4.15, R_{2}=0.95, \omega=0.3 ; E$ is explosive energy, $E=7 \mathrm{Gpa}$; TNT is selected as explosive in this model, explosive velocity $v_{d}=6930 \mathrm{~m} / \mathrm{s}$, density $\rho_{0}=1630 \mathrm{~kg} / \mathrm{m}^{3}$, explosion pressure $P_{c j}=0.21 \mathrm{Gpa}$.

The blasted rock mass is granite, and the constitutive model of rock mass is isotropic-elastic-plastic material. Its state equation is as follows:

$$
p=\frac{\rho_{o} c^{2} v\left[1+(1-\mu / 2) v-\alpha v^{2} / 2\right]}{\left[1-\left(s_{1}-1\right) v+s_{2} v^{2} /(v+1)-s_{2} v^{3} /(v+1)^{2}\right]^{2}}+(\mu+\alpha v) E
$$

where $s_{1}=1.5, s_{2}=0, s_{3}=0, \gamma=2.0, \alpha=0.5, \quad v=1 / v_{o}-1\left(v_{o}\right.$ is relative volume); the material parameters of granite are: Young's modulus $E=66 \mathrm{Gpa}$, Poisson's ratio $\mu=0.23$, density $\rho_{0}=2700 \mathrm{~kg} / \mathrm{m}^{3}$, wave velocity $c=5268 \mathrm{~m} / \mathrm{s}$, shear modulus $G=23 \mathrm{Gpa}$, hardening parameter $n=0.25$.

The mesh arrangement of cut holes is shown in Figure 2. Millisecond blasting of different parameters of charge of the first section, maximum charge of one section, number of sections and delay interval were simulated between 10 boreholes by setting different initiation sequence.

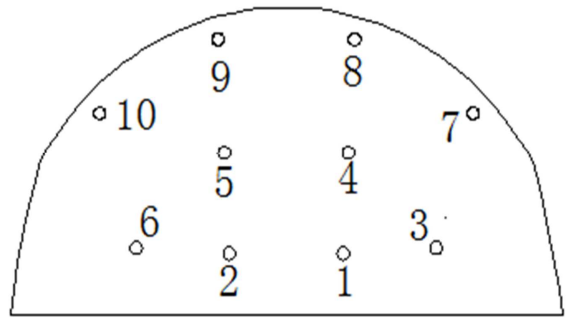

Figure 2. Diagram of mesh arrangement of cutting holes.

Table 1 is a sample of 13 groups of blasting vibration data obtained by using the numerical model to simulate various working conditions.

\begin{tabular}{|c|c|c|c|c|c|c|c|c|}
\hline \multirow[b]{2}{*}{ No. } & \multicolumn{3}{|c|}{ Relevant Variables } & \multicolumn{5}{|c|}{ System Characteristic Variables } \\
\hline & $\begin{array}{l}X_{1} \\
(\mathrm{~kg}) \\
\end{array}$ & $\begin{array}{l}X_{2} \\
(\mathrm{~kg}) \\
\end{array}$ & $\begin{array}{l}X_{3} \\
(\mathrm{~ms}) \\
\end{array}$ & $X_{4}$ & $\begin{array}{l}Y_{1} \\
(\mathrm{~cm} / \mathrm{s})\end{array}$ & $\begin{array}{l}Y_{2} \\
(\mathrm{~Hz})\end{array}$ & $\begin{array}{l}Y_{3} \\
(\mathrm{~cm} / \mathrm{s})\end{array}$ & $\begin{array}{l}Y_{4} \\
(\%)\end{array}$ \\
\hline 1 & 5.1 & 5.1 & 35 & 2 & 7.25 & 16 & 0.91 & 87.4 \\
\hline 2 & 3.4 & 3.4 & 35 & 3 & 7.25 & 16 & 0.95 & 86.9 \\
\hline 3 & 6.8 & 6.8 & 35 & 2 & 7.4 & 15.99 & 1.17 & 84.2 \\
\hline 4 & 0.85 & 6.8 & 35 & 3 & 4.05 & 27.99 & 0.77 & 80.9 \\
\hline 5 & 1.7 & 3.4 & 35 & 3 & 5.26 & 83.96 & 0.86 & 83.7 \\
\hline 6 & 1.7 & 6.8 & 35 & 2 & 4.89 & 31.98 & 0.76 & 84.5 \\
\hline 7 & 1.7 & 3.4 & 35 & 4 & 4.89 & 31.98 & 0.84 & 82.8 \\
\hline 8 & 5.1 & 5.1 & 0 & 2 & 13.0 & 62.83 & 1.51 & 88 \\
\hline 9 & 5.1 & 5.1 & 8 & 2 & 8.36 & 10.95 & 1.21 & 85 \\
\hline 10 & 5.1 & 5.1 & 15 & 2 & 9.66 & 91.95 & 0.99 & 0.99 \\
\hline 11 & 5.1 & 5.1 & 35 & 2 & 6.67 & 91.95 & 1.07 & 1.07 \\
\hline 12 & 5.1 & 5.1 & 50 & 2 & 6.67 & 59.97 & 1.14 & 1.14 \\
\hline 13 & 5.1 & 5.1 & 70 & 2 & 6.67 & 63.97 & 1.09 & 1.09 \\
\hline
\end{tabular}

Table 1. Sample of blasting vibration data.

\subsection{Calculation of Grey Relational Degree}

In order to determine the positive and negative relation between the related variables and the system characteristic variables, the relation should be transformed by axial symmetry method. Explosion charge is negatively related with main frequency, number of sections and delay interval is negatively related with particle peak velocity, the relevant variables are positively related with average combined velocity and velocity attenuation rate. Initial data are usually transformed into dimensionless data with similar order of magnitude in the process of grey relational analysis, and the negative relation factors are transformed into positive relation factors. Based on the sample data in Table 1, the grey relational matrix of the factors affecting blasting vibration was calculated with method of grey relational analysis, as shown in Table 2.

Table 2. Grey relational matrix of the factors affecting blasting vibration.

\begin{tabular}{lllll}
\hline Relational Degree & $\boldsymbol{X}_{\boldsymbol{1}}$ & $\boldsymbol{X}_{\mathbf{2}}$ & $\boldsymbol{X}_{\mathbf{3}}$ & $\boldsymbol{X}_{\boldsymbol{4}}$ \\
\hline$Y_{1}$ & 0.7792 & 0.7619 & 0.7252 & 0.6878 \\
$Y_{2}$ & 0.6857 & 0.7337 & 0.6057 & 0.5928 \\
$Y_{3}$ & 0.7039 & 0.7410 & 0.8493 & 0.7432 \\
$Y_{4}$ & 0.7281 & 0.7559 & 0.7563 & 0.6605 \\
$\sum$ & 2.8969 & 2.9925 & 2.9365 & 2.6843 \\
\hline
\end{tabular}




\subsection{Analysis on Influencing Factors of Blasting Vibration Caused by Shallow Tunnel Excavation}

Referring to the grey relational matrix of the influencing factors of blasting vibration in Table 2, superiority ranks analysis of the related variables were carried out, and the following conclusions can be drawn:

The superiority ranks of influencing factors of blasting vibration caused by shallow tunnel excavation in the order of high to low are: maximum charge of one section, charge of the first section, delay interval and number of sections; there is no optimal factor, and the maximum charge of one section is the quasi-optimal factor.

The superiority ranks of influencing factors of particle peak velocity for blasting vibration in the order of high to low are: charge of the first section, maximum charge of one section, delay interval and number of sections; combining with the comparative analysis of particle peak velocity of different charge of the first section in Table 1, it is shown that the charge of the first section is the main factor affecting the peak velocity of blasting vibration.

The superiority ranks of influencing factors of main frequency for blasting vibration in the order of high to low are: maximum charge of one section, charge of the first section, delay interval and number of sections; the maximum charge of one section is the main factor affecting the main frequency of blasting vibration.

The superiority ranks of influencing factors of average combined velocity and velocity attenuation rate for blasting vibration in the order of high to low are: delay interval, maximum charge of one section, charge of the first section and number of sections; delay interval is the optimal factor for the two system characteristic variables, which is also the main factor affecting the duration of blasting vibration. In the superiority ranks of the factors affecting the duration, the ranks of the factors affecting the average combined velocity and the velocity attenuation ratio show consistency, which indicates that the two parameters selected to represent the duration of blasting vibration are reasonable.

As one of the influencing factors of blasting vibration, the number of sections is not a quasi-optimal factor and the main influencing factor. Excessive increase of the number of initiation sections is not an effective means to control the blasting vibration effect in millisecond blasting.

\section{Control Measures of Blasting Vibration Caused by Shallow Tunnel Excavation}

Based on the above conclusions, the following measures are suggested to control the blasting vibration caused by shallow tunnel excavation:

To reduce the charge of the first section. Because the number of free surfaces is small and the initiation energy of the first section is too concentrated, the blasting vibration caused is large. At the same time, due to the serious shortage of compensation space after blasting, blasting rock is subject to greater resistance when it disperses, and the blasting effect is poor. Therefore, reducing the charge of the first section can effectively control the blasting vibration intensity and improve the blasting effect.

To reduce the maximum charge of one section. The maximum charge of one section is the main factor affecting blasting vibration. Therefore, under the condition of constant total blasting charge, the blasting vibration intensity can be effectively reduced by properly increasing the initiation sections and reducing the maximum charge of one section.

To set up rational delay interval. For millisecond blasting of shallow tunnel, reasonable delay interval is more effective than increasing the number of sections. Cutting holes can be set as jump-off, which can prolong the interval between sections properly and prevent the superposition of blasting seismic waves, so as to achieve the purpose of vibration reduction. Cutting holes can be set as jump-off blasting holes, which can prolong the interval between sections and prevent the superposition of blasting vibration waves, so as to achieve the purpose of vibration reduction.

\section{Conclusion}

The sample data of blasting vibration were obtained by establishing finite element model of cut blasting in shallow tunnel. Based on the sample data, the relational degree analysis was carried out for influencing factors of blasting vibration caused by shallow tunnel excavation with method of grey relational analysis. The analysis results show that: among the four relevant influencing factors, there is no optimal factor, and the maximum charge of one section is the quasi-optimal factor; the charge of the first section is the main factor affecting the peak velocity of blasting vibration; the maximum charge of one section is the main factor affecting the main frequency of blasting vibration; the delay interval the main factor affecting the duration of blasting vibration.

Based on the conclusion of grey relational analysis, the measures to control blasting vibration caused by shallow tunnel excavation were put forward, such as reducing the charge of the first section, reducing the maximum charge of one section and rationally setting up the delay interval.

There are many factors affecting blasting vibration caused by shallow tunnel construction. Only some of the factors were considered based on the numerical test model in this paper. It is suggested that field tests can be carried out to further analyze the influence of other factors such as number of free surfaces, hole network parameters and geological conditions.

\section{Acknowledgements}

The authors would like to acknowledge the financial support from the National Natural Science Foundation of China (51504082 and 51874123) and the Science and Technology Research Planning Project of Henan Province, China (152102310331). 


\section{References}

[1] P. K. Singh. Blast vibration damage to underground coal mines from adjacent open-pit blasting [J]. International Journal of Rock Mechanics and Mining Sciences, 2002, 39 (8): 959-973.

[2] Qihu Qian, Shihai Chen. Blasting vibration effect [J]. Blasting, 2004, 21 (2): 1-5.

[3] Chengyu Xie, Zhouquan Luo, Nan Jia, Lixin Xiong, Guihai Cheng. Dynamic effects of open blasting vibration on adjacent buildings and measures for vibration reduction [J]. Journal of Vibration and Shock, 2013, 32 (13): 187-193.

[4] Ranjan Kumar, Deepankar Choudhury, Kapilesh Bhargava. Determination of blast-induced ground vibration equations for rocks using mechanical and geological properties [J]. Journal of Rock Mechanics and Geotechnical Engineering, 2016, 8 (3): 341-349.

[5] Wenzhuo Cao, Xibing Li, Ming Tao, et al. Vibrations induced by high initial stress release during underground excavations [J]. Tunnelling and Underground Space Technology, 2016, 53: 78-95.

[6] Shihai Chen, Shuaiwei Hu, Zihua Zhang, et al. Propagation characteristics of vibration waves induced in surrounding rock by tunneling blasting [J]. Journal of Mountain Science, 2017, 14 (12): 2620-2630.

[7] Nan Jiang, Tan Gao, Chuanbo Zhou, et al. Effect of excavation blasting vibration on adjacent buried gas pipeline in a metro tunnel [J]. Tunnelling and Underground Space Technology, 2018, 81: 590-601.

[8] Mesec J, Kovac I, Soldo B. Estimation of particle velocity based on blast event measurements at different rock units [J], Soil Dynamics and Earthquake Engineering, 2010, 30 (10): 1004-1009.

[9] Mingsheng Zhao, Kaishui Liang, Benwei Li. Influence of deck charge on time-frequency characteristics of a blasting vibration signal [J]. Journal of Vibration and Shock, 2012, 31 (7): 85-88.

[10] D. P. Blair. Blast vibration dependence on charge length, velocity of detonation and layered media [J]. International Journal of Rocks Mechanics and Mining Sciences, 2014, 65: 29-39.

[11] Junru Zhou, Wenbo Lu, Le Zhang, Ming Chen, Peng Yan. Attenuation of vibration frequency during propagation of blasting seismic wave [J]. Chinese Journal of Rock Mechanics and Engineering, 2014, 33 (11): 2171-2178.

[12] Zhengzheng Wang, Yangsheng Zhang. Numerical analysis for blasting vibration characteristics in tunnel excavation based on ALE algorithm [J]. Journal of Dalian University of Technology, 2017, 57 (3): 279-284.

[13] Zhiwen Li, Jianchun Li, Shengnan Hong, Haibo Li, Guokai Zhang. Theoretical analysis of blasting vibration zone considering viscous effect [J]. Journal of Vibration and Shock, 2018, 37 (17): 108-114.

[14] Sifeng Liu, Tianbang Guo, Yaoguo Dang. Grey system theory and its application [M]. Beijing: Science Press, 2013: 1-30.

[15] Zijun Li. Advance of study on application of grey system theory in China during 1992-2001[J]. Systems Engineering, 2003, 21 (5): 8-12.

[16] Dang Luo, Sifeng Liu. Study on the method for grey incidence decision-making [J]. Chinese Journal of Management Science, 2005, 13 (1): 101-106.

[17] Julong Deng. Basic method of grey system [M]. Wuhan: Huazhong University of Technology Press, 1988: 17-28. 